\title{
CARACTERÍSTICAS MORFOLÓGICAS Y PRODUCCIÓN DE ALOÍNA DE PLANTAS POLIPLOIDES DE Aloe vera (L.) Burm. F. (ASPHODELACEAE)
}

\author{
Tamara Molero Paredes ${ }^{1}$ y Maribel Viloria ${ }^{2}$
}

\begin{abstract}
RESUMEN
Aloe vera es una especie medicinal que ha alcanzado gran demanda por las propiedades de sus metabolitos secundarios, como la aloína. Sin embargo, la lenta tasa de crecimiento de la planta y la baja cantidad de este metabolito en su biomasa ha hecho necesaria la búsqueda de estrategias que mejoren la calidad de la planta. El objetivo de este trabajo fue determinar las características morfológicas y el contenido de aloína in vitro de plantas poliploidizadas de A. vera provenientes de siete poblaciones de Venezuela. Los valores de longitud y ancho foliar, así como el número de hojas, peso seco, concentración y producción de aloína fueron mayores en las plantas poliploides de todas las poblaciones en comparación a los controles diploides. De igual manera, se encontraron diferencias altamente significativas entre las poblaciones poliploides. Las plantas de la localidad de Carazao manifestaron los mayores valores promedio de concentración (7,69 \%) y producción de aloína (0,169 g por 100 g de peso seco) como respuesta a la duplicación cromosómica artificial. La explotación de estos nuevos genotipos podría mejorar el cultivo de la sábila a nivel comercial e industrial, a la vez que los nuevos conocimientos destacan el potencial de la poliploidía inducida como un medio rápido para mejorar características morfológicas y la producción de metabolitos secundarios en cultivos. Palabras clave adicionales: In vitro, morfología vegetal, poliploidía, sábila
\end{abstract}

\begin{abstract}
Morphological characteristics and aloin production in polyploid plants of Aloe vera (L.) Burm. F. (Asphodelaceae) Aloe vera is a medicinal species that has been in great demand for the properties of its secondary metabolites, such as aloin. However, the slow plant growth rate and low quantity of this metabolite in its biomass has made it necessary to search for strategies that improve the quality of the plant. The objective of this work was to determine the morphological characteristics and aloin content in polyploidized aloe vitroplants from seven populations in Venezuela. The values of height, number of leaves and width of the leaf, dry mass, concentration and aloin production were higher in polyploid plants of all populations compared to diploid controls. Similarly, highly significant differences were found between polyploid populations. The plants from locality of Carazao showed the highest mean values of aloin concentration (7.69 \%) and production (0.169 g per $100 \mathrm{~g}$ dry weight) in response to artificial chromosomal duplication. The growing of these new genotypes could improve the utilization of aloe at a commercial and industrial level, and the new knowledge shows the potential of induced polyploidy as a rapid means to improve morphological characteristics and production of secondary metabolites in crops.
\end{abstract}

Additional keywords: Aloe plant, in vitro, plant morphology, polyploidy

\section{INTRODUCCIÓN}

Una de las aplicaciones de la técnica de inducción de poliploidía en plantas, es lograr el aumento de la biomasa y la cantidad de metabolitos secundarios. Ejemplo de ello son los casos de las plantas de Artemisia annua (De Jesús y Weathers, 2003; Zanmaria, 2016), Taxus chinensis (Li et al., 2008) Citrus reticulata (Tan et al., 2019) e híbridos del género Populus (Cheng et al., 2019) en las cuales lograron obtener en algunos casos tetraploides $y$ en otros triploides, con aumento de las cantidades de artemisina, taxol, ácido ascórbico y flavonoides, respectivamente, que son los metabolitos secundarios más conocidos en estas especies. En Valeriana wallichii se generó la producción de nuevos compuestos en las plantas, ausentes en sus

Recibido: Mayo 22, 2020

Aceptado: Octubre 15, 2020

${ }^{1}$ Coordinación de Biología. Facultad de Humanidades. Universidad Adventista Dominicana. República Dominicana

${ }^{2}$ Dpto. de Biología. Facultad de Humanidades y Educación. Universidad del Zulia. Venezuela.

e-mail: tamaramolero@unad.edu.do 
parentales.

En el caso de $A$. vera, su principal metabolito secundario es la aloína, el cual es considerado como el principio activo de la planta, se produce en mayor concentración y ha sido empleado como un marcador taxonómico de donde deriva el nombre del género Aloe. Este compuesto, en conjunto con una cantidad alta de fitoquímicos y antioxidantes que se encuentran en la planta, le confiere las propiedades medicinales y nutritivas que posee (Pandey y Singh, 2016; Cardarelli et al, 2017).

Químicamente, la aloína (10-C-ßglucopiranósido de aloe-emodin-antrona) es un metabolito considerado como un C-heterósido, producto de la combinación de una antraquinona simple (genina) con un azúcar (glucosa) y se extrae de fuentes naturales como una mezcla de dos diastereoisómeros, llamados aloína-A (también conocida como barbaloína) y aloína-B (o isobarbaloína) (Martínez et al., 2017).

De esta planta no solamente se obtiene la aloína en polvo, sino también una gama de productos y subproductos que son empleados en la industria medicinal, alimentaria y cosmetológica cuya producción se ve limitada por una serie de condiciones como la lenta tasa de propagación en campo, la baja concentración de metabolitos secundarios extraídos de las plantaciones comerciales y la incidencia de enfermedades (Piña y Chirino, 2008). De allí ha surgido la necesidad de buscar alternativas que aumenten la tasa de propagación de las plantas sanas y de los productos derivados de ella, evitando la aplicación de agroquímicos en el cultivo, puesto que es uno de los estándares exigidos por sociedades internacionales como el ASC (Aloe Science Council) y la Unión Europea (CEE Directiva del Consejo 88/388). El cultivo in vitro combinado con las técnicas de poliploidización ha dado buenos resultados en la obtención de especies sanas con mejores condiciones fenotípicas (Manzoor et al., 2019).

Varios investigadores han trabajado sobre la estandarización de la técnica para la inducción de poliploidía en $A$. vera y sus efectos en las características morfológicas de la planta (Ren et al., 2007; Molero y Matos, 2008; Molero et al., 2018). Algunas de estas investigaciones indican que las plantas han manifestado el fenómeno "gigas", es decir, mayor crecimiento de algunas partes de la planta; sin embargo, hasta ahora no se ha medido el efecto de la duplicación cromosómica sobre la concentración de sus metabolitos secundarios. Por ello, el objetivo de esta investigación fue cuantificar la producción de aloína y determinar las características morfológicas en plantas poliploides de Aloe vera (L.) de forma comparativa con sus equivalentes diploides.

\section{MATERIALES Y MÉTODOS}

Obtención del material. Para realizar esta investigación se seleccionaron al azar 30 vitroplantas poliploides ( $2 \mathrm{n}=28$ cromosomas) de siete poblaciones (1-Cumarebo, 2-Carazao, 3Adaure, 4-Tamare, 5-Caramón, 6-Mayales, 7Puertos de Altagracia) del occidente de Venezuela. Las vitroplantas se encontraban en el Laboratorio de Biotecnología de la Facultad de Agronomía de la Universidad del Zulia (Venezuela), las cuales habían sido previamente establecidas, multiplicadas y tratadas con colchicina para la inducción artificial de la poliploidía en condiciones de laboratorio según los métodos descritos por Molero et al. (2018). De igual manera se emplearon 30 vitroplantas diploides como control ( $2 n=14$ cromosomas), para conformar un arreglo factorial de los tratamientos con dos tipos de ploidía y siete clones (poblaciones de procedencia).

Estudio de las características morfológicas y producción de materia fresca y seca. Las vitroplantas poliploides y diploides (control) fueron trasladadas al área de aclimatación del Laboratorio de Biotecnología, en el cual fueron extraídas cuidadosamente de los frascos con medios de cultivo y cultivadas en envases plásticos de $200 \mathrm{~cm}^{3}$ que contenían arena y abono orgánico (50:50) previamente desinfectados. Al inicio, las plántulas presentaban un promedio de 4 hojas y $12 \mathrm{~cm}$ de altura. En este lugar se mantuvieron durante 3 meses en plena sombra, con temperatura de laboratorio $\left(28 \pm 1{ }^{\circ} \mathrm{C}\right)$ y un riego semanal.

Transcurrido este tiempo, se procedió a medir en todas las plantas las características morfológicas de longitud media de la hoja, ancho medio y número de hojas.

De igual manera se registró el peso seco, para lo cual las plantas completas fueron previamente fraccionadas y homogeneizadas en una licuadora. 
El contenido se almacenó en bolsas herméticas en un congelador y posteriormente fue liofilizado (Labconco Lyph-Lock 6) durante 72 horas. Este mismo material se empleó para la determinación del contenido de aloína.

Cuantificación de la aloína. La concentración de este compuesto fenólico se determinó mediante cromatografía líquida de alta resolución (HPLC). Para ello, las muestras liofilizadas de las plantas de cada población fueron enviadas al Laboratorio de Cromatografía de la Facultad de Agronomía de la Universidad del Zulia, Venezuela, donde se realizó la extracción etanólica de la aloína según el protocolo utilizado por Park et al. (1998) y la separación cromatográfica según la metodología reportada por Molero et al. (2016). Se empleó un cromatógrafo líquido de alta resolución (Shimadzu modelo 10A), equipado con un detector UV (SPD-10A), operado a $293 \mathrm{~nm}$. Como fase móvil se empleó metanol:agua $(50: 50 \% \mathrm{v} / \mathrm{v})$ a un flujo de $1,0 \mathrm{~mL} \cdot \mathrm{min}^{-1} \mathrm{y}$ como fase estacionaria una columna Zorbax SB-C18 (250 mm x 4,60 mm x $\mu \mathrm{m}$ de octadesilsilano). Se empleó un estándar puro de aloína Sigma con un $97 \%$ de pureza.

La concentración de aloína se determinó a partir del área del triángulo formado en la línea pico del cromatograma del HPLC, mientras que la producción de aloína se obtuvo a partir del valor de su concentración con relación al peso seco.
Los resultados se evaluaron aplicando las técnicas de la estadística inferencial mediante análisis de varianza (ANOVA) y pruebas de medias de Tukey empleando el paquete estadístico SPSS versión 20.

\section{RESULTADOS}

Al observar los resultados de las variables estudiadas en esta investigación entre las plantas diploides y poliploides se nota que los datos de varias características fueron diferentes entre ambos grupos. Es el caso de la longitud foliar, ancho foliar y número de hojas (Cuadro 1), peso seco (Cuadro 2), y concentración y producción de aloína (Cuadro 3), donde las plantas poliploides casi siempre presentaron los valores más altos. Las diferencia en la longitud foliar encontrada entre diploides y poliploides fue la característica más distintiva en los rasgos morfológicos.

Para la variable número de hojas no se detectaron diferencias entre algunas de las poblaciones estudiadas, aunque sí fueron muy evidentes las diferencias existentes dentro de cada una de dichas poblaciones (Cuadro 1).

En el Cuadro 1 se visualizan las características morfológicas de las plantas de $A$. vera poliploides y diploides provenientes de diferentes poblaciones, en la cual se aprecia que casi todos los valores de las variables en las plantas control fueron inferiores a los poliploides.

Cuadro 1. Características morfológicas de plantas de $A$. vera diploides y poliploides

\begin{tabular}{lcccccc} 
Población & \multicolumn{2}{c}{ Longitud foliar $(\mathrm{cm})$} & \multicolumn{2}{c}{ Número de hojas } & \multicolumn{2}{c}{ Ancho foliar $(\mathrm{cm})$} \\
\cline { 2 - 7 } (Clon) & Diploide & Poliploide & Diploide & Poliploide & Diploide & Poliploide \\
\hline 1 Cumarebo & 19,43 Bab & $33,80 \mathrm{Ac}$ & $6,46 \mathrm{Aab}$ & $7,53 \mathrm{Aab}$ & $1,80 \mathrm{Ba}$ & $3,17 \mathrm{Acd}$ \\
2 Carazao & $19,56 \mathrm{Bab}$ & $40,55 \mathrm{Ab}$ & $4,52 \mathrm{Bb}$ & $7,44 \mathrm{Aab}$ & $1,84 \mathrm{Ba}$ & $4,26 \mathrm{Aab}$ \\
3 Adaure & $22,95 \mathrm{Bab}$ & $48,38 \mathrm{Aa}$ & $6,04 \mathrm{Aab}$ & $5,50 \mathrm{Ab}$ & $1,77 \mathrm{Ba}$ & $3,94 \mathrm{Abc}$ \\
4 Tamare & $21,35 \mathrm{Bab}$ & $29,82 \mathrm{Ac}$ & $6,40 \mathrm{Bab}$ & $8,27 \mathrm{Aa}$ & $1,78 \mathrm{Ba}$ & $3,18 \mathrm{Acd}$ \\
5 Caramón & $25,00 \mathrm{Ba}$ & $40,20 \mathrm{Ab}$ & $8,33 \mathrm{Aa}$ & $8,66 \mathrm{Aa}$ & $1,87 \mathrm{Ba}$ & $3,03 \mathrm{Ad}$ \\
6 Mayales & $16,56 \mathrm{Bb}$ & $26,95 \mathrm{Ad}$ & $4,63 \mathrm{Ab}$ & $6,42 \mathrm{Aab}$ & $3,72 \mathrm{Ba}$ & $3,05 \mathrm{Ad}$ \\
7 P. Altagracia & $14,95 \mathrm{Bb}$ & $44,83 \mathrm{Aab}$ & $6,13 \mathrm{Bab}$ & $8,75 \mathrm{Aa}$ & $2,00 \mathrm{Ba}$ & $3,74 \mathrm{Aabc}$ \\
\hline
\end{tabular}

Valores con distintas letras mayúsculas entre columnas y minúsculas entre filas indican diferencias significativas según la prueba de Tukey $(P \leq 0,01)$

Al comparar los resultados encontrados para la longitud foliar, el análisis de varianza indica que hubo diferencias altamente significativas $(P \leq 0,01)$ entre las poblaciones tanto controles como poliploides, siendo las plantas de Caramón las que se destacaron con el mayor promedio $(25,00 \mathrm{~cm})$ entre las plantas control, mientras que las de Adaure fueron las que lograron desarrollar el mayor promedio $(48,38 \mathrm{~cm})$ entre las plantas poliploides.

Al analizar la variable número de hojas, los resultados demostraron que hubo una diferencia 
significativa $(P \leq 0,05)$ entre las poblaciones control, siendo las plantas de Caramón las que desarrollaron mayor número promedio de hojas $(8,33)$, y entre las plantas poliploides, hubo una diferencia altamente significativa $(P \leq 0,01)$ destacándose las plantas de Puertos de Altagracia $(8,75)$, Caramón $(8,66)$ y Tamare $(6,40)$ por presentar valores altos.

Con respecto al ancho foliar, el análisis de varianza indica que no hubo diferencias significativas entre las poblaciones de las plantas control, pero si las hubo entre los poliploides, manifestándose diferencias altamente significativas $(P \leq 0,01)$, donde se destacan las pertenecientes a la población Carazao. Al comparar los valores de ancho de la hoja entre las plantas diploides y poliploides, puede notarse que éstas últimas duplicaron su valor en relación con los controles, lo que significa que produjeron mayor cantidad de biomasa.

Es importante destacar que el número de hojas encontrado en las plantas poliploides procedentes de Adaure y el ancho foliar en las plantas de Mayales resultó inferior que en el caso de las plantas diploides, lo cual corresponde a una respuesta atípica, diferente al resto de las evaluaciones, y para lo cual no se sugiere alguna explicación pertinente.

En el Cuadro 2 se muestran los resultados correspondientes a la separación de medias para la producción de peso seco en las plantas de las siete poblaciones en estudio. Resalta que, en todos los casos, los valores promedios en los controles diploides fueron inferiores a los poliploides. Asimismo, se destaca que el clon proveniente de la población de Adaure fue el que presentó el mayor peso seco promedio independientemente de la ploidía.

Cuadro 2. Producción de peso seco en las plantas de Aloe vera diploides y poliploides de diferentes poblaciones del occidente de Venezuela

\begin{tabular}{lcc}
\hline \multirow{2}{*}{ Población (Clon) } & \multicolumn{2}{c}{ Peso seco (g por planta) } \\
\cline { 2 - 3 } 1 (Cumarebo) & Diploide & Poliploide \\
2 (Carazao) & $1,26 \mathrm{Bb}$ & $2,00 \mathrm{Abc}$ \\
3 (Adaure) & $1,18 \mathrm{Bbc}$ & $2,20 \mathrm{Aab}$ \\
4 (Tamare) & $1,76 \mathrm{Ba}$ & $2,50 \mathrm{Aa}$ \\
5 (Caramón) & $0,98 \mathrm{Bc}$ & $2,10 \mathrm{Aab}$ \\
6 (Mayales) & $1,09 \mathrm{Bbc}$ & $1,70 \mathrm{Ac}$ \\
7 (P. Altagracia) & $0,91 \mathrm{Bc}$ & $1,20 \mathrm{Ad}$ \\
\hline
\end{tabular}

Valores con distintas letras mayúsculas entre columnas y minúsculas entre filas indican diferencias significativas según la prueba de Tukey $(P \leq 0,05)$

En cuanto a la característica de peso seco para las plantas de $A$. vera diploides y poliploides, el Cuadro 2 muestra que el valor de peso seco en los controles fue menor con respecto a los poliploides. Hubo diferencias estadísticas entre las poblaciones control, siendo los clones provenientes de las poblaciones de Adaure y Puertos de Altagracia los que presentaron los valores mayores por planta (1,76 y 1,27 g, respectivamente). Con relación a las plantas poliploides, se formaron cinco grupos en función a las localidades en estudio, siendo el clon de la población de Adaure el que presentó el valor mayor (2,50 g por planta).

En cuanto a la concentración de aloína, se puede observar en el Cuadro 3 que las plantas diploides presentan menor concentración de aloína comparativamente con los poliploides.
De igual manera, se observan diferencias altamente significativas $(P \leq 0,01)$ y significativas $(P<0,05)$ entre las poblaciones de plantas diploides y poliploides respectivamente. En el caso de las plantas control, las provenientes de la localidad de Carazao se destacaron con un valor máximo de 3,72\%, seguidas por las de la población de Caramon con 3,55 \% de aloína.

En las plantas poliploides, se destacaron las procedentes de Carazao y Adaure con una concentración del metabolito de 7,69 y 5,7 \%, respectivamente.

Al determinar la producción total de aloína, nuevamente los valores encontrados para las plantas control fueron inferiores a las plantas poliploides. En este sentido, el análisis de la varianza refleja que dentro de las plantas control, 
se forman cinco grupos de acuerdo con la población, encontrándose los valores máximos en las plantas de la población de Adaure $(0,055 \mathrm{~g}$ por $100 \mathrm{~g}$ ), mientras que el resto de las localidades tuvieron valores inferiores.

En cuanto a la producción de aloína en las plantas poliploides, la prueba de medias reveló que entre las poblaciones en estudio se formaron cinco grupos, donde las plantas de Carazao y Adaure fueron las que respondieron mejor a la poliploidización, con 0,169 y 0,142 g por 100 g, respectivamente.

Cuadro 3. Concentración de aloína en las plantas de Aloe vera diploides y poliploides de diferentes localidades del occidente de Venezuela

\begin{tabular}{lcccc}
\hline \multicolumn{2}{c}{ Población (Clon) } & \multicolumn{2}{c}{ Concentración de aloína (\%) } & \multicolumn{2}{c}{ Producción de aloína (g por 100 g) } \\
\hline & Diploide & Poliploide & Diploide & Poliploide \\
\hline 1 (Cumarebo) & $3,28 \mathrm{Bbc}$ & $4,26 \mathrm{Abc}$ & $0,041 \mathrm{Bc}$ & $0,085 \mathrm{Acd}$ \\
2 (Carazao) & $3,72 \mathrm{Ba}$ & $7,69 \mathrm{Aa}$ & $0,047 \mathrm{Bb}$ & $0,169 \mathrm{Aa}$ \\
3 (Adaure) & $3,15 \mathrm{Bbc}$ & $5,7 \mathrm{Aab}$ & $0,055 \mathrm{Ba}$ & $0,142 \mathrm{Ab}$ \\
4 (Tamare) & $2,17 \mathrm{Be}$ & $4,74 \mathrm{Abc}$ & $0,021 \mathrm{Be}$ & $0,099 \mathrm{Abc}$ \\
5 (Caramón) & $3,55 \mathrm{Bb}$ & $4,46 \mathrm{Abc}$ & $0,038 \mathrm{Bd}$ & $0,075 \mathrm{Acd}$ \\
6 (Mayales) & $3,02 \mathrm{Bc}$ & $3,87 \mathrm{Ac}$ & $0,027 \mathrm{Be}$ & $0,046 \mathrm{Ae}$ \\
7 (P. Altagracia) & $2,88 \mathrm{Bd}$ & $3,52 \mathrm{Ac}$ & $0,036 \mathrm{Bd}$ & $0,059 \mathrm{Ae}$ \\
\hline
\end{tabular}

Valores con distintas letras mayúsculas entre columnas y minúsculas entre filas indican diferencias significativas según la prueba de Tukey $(P \leq 0,01)$

En la Figura 1 se muestra el cromatograma de la planta poliploide de la población de Carazao, la cual presentó la concentración más alta de aloína. Como puede observase en la gráfica, a los 10 minutos se produjo la elución de la aloína en la columna del cromatógrafo y el cálculo del área del pico se corresponde con una concentración de $7,69 \%$.

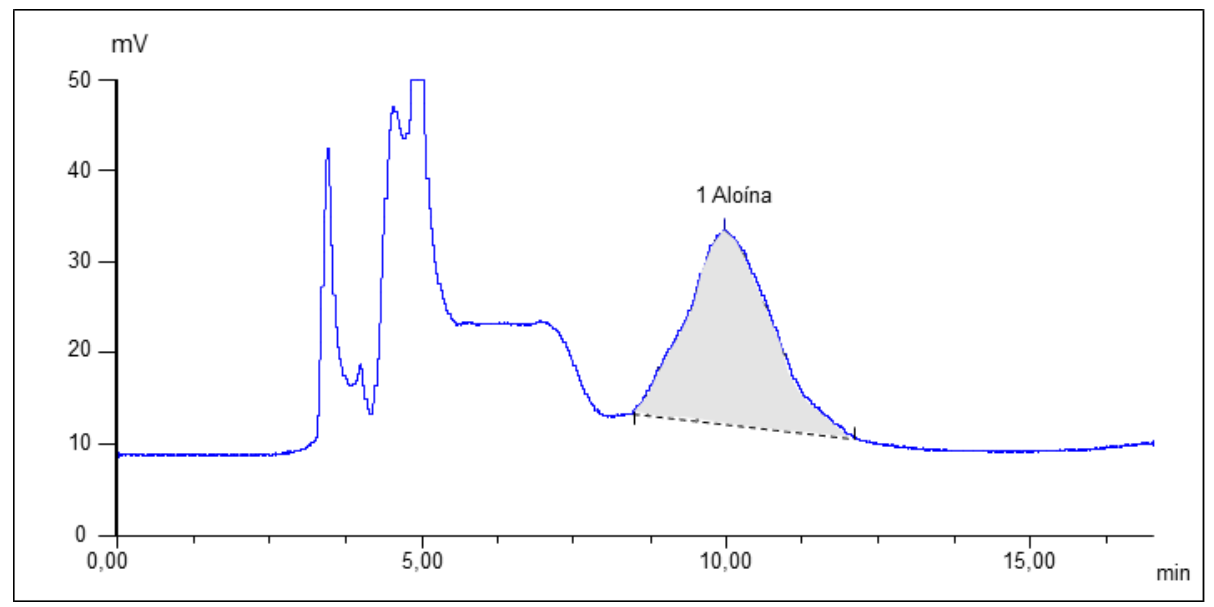

Figura 1. Cromatograma de HPLC del extracto etanólico de una vitroplanta de Aloe vera poliploide de la localidad de Carazao donde se muestra la máxima concentración registrada de aloína (equivalente a 7,69 \% del metabolito según el área del triángulo generado)

\section{DISCUSIÓN}

Una de las características principales de los poliploides es el desarrollo de estructuras y órganos de mayor tamaño, fenómeno que se conoce como "gigantismo" (Pierce, 2009). Los valores obtenidos en nuestro estudio para la longitud y ancho foliar, así como el número de hojas y el peso seco ponen de manifiesto que la duplicación cromosómica incrementó la cantidad de biomasa en la planta, así como también influyó sobre la concentración y producción de aloína.

Los trabajos realizados por Wang et al. (2001) y Ren et al. (2007) demostraron que las plantas de 
A. vera poliploidizadas artificialmente in vitro manifestaron expresiones de gigantismo en las hojas mostrando ser más gruesas, grandes, con un color verde más oscuro en comparación a las plantas control empleadas en esa investigación. Los resultados de estos estudios son compatibles con los encontrados en nuestra investigación.

La literatura en el campo del mejoramiento genético describe las consecuencias del proceso de poliploidía en el fenotipo de las plantas de manera natural o inducida (Cubero, 2003; Pierce, 2009), pero actualmente las investigaciones están dirigidas a tratar de explicar la base metabólica subyacente al vigor de la poliploidización. En este sentido, Doyle y Coate (2019) explican que el aumento del tamaño de la célula por el proceso de poliploidía, juega un papel clave en los cambios fisiológicos y de desarrollo asociados con la duplicación del genoma. Los autores plantean la hipótesis de que el tamaño celular, el volumen nuclear y la duración del ciclo celular se encuentran entre un conjunto de caracteres "nucleotípicos" que son afectados por el proceso de poliploidización y esto influye en todo el funcionamiento celular, incluyendo la síntesis de proteínas y metabolitos primarios y secundarios, que se manifiestan en la planta como cambios fenotípicos influenciados por la cantidad de ADN en masa, independientemente del contenido genético. Por su parte, Corneillie et al. (2019) mostraron que los poliploides se desarrollan más rápidamente en comparación con los diploides y, al caracterizar la pared celular, observaron que el aumento en la ploidía se correlacionó negativamente con el contenido de lignina y celulosa, y positivamente con el contenido de polisacáridos de la matriz (hemicelulosa), lo que podría explicar la mayor producción de biomasa en una planta poliploide.

De igual manera, Cheng et al. (2019) realizaron un estudio sobre mapeo y cuantificación de los metabolitos y su relación con el vigor del crecimiento en plantas de Populus poliploides y encontraron que la diversidad y cantidad de metabolitos producidos estaban en relación directa con la ploidía, y en todos los casos, los aminoácidos, carbohidratos y las sustancias derivadas del metabolismo secundario, fueron los principales metabolomas que se asociaron positivamente con las altas tasas y vigor del crecimiento.
La obtención de plantas poliploides para incrementar los niveles de metabolitos secundarios se ha hecho con el objetivo de aumentar compuestos importantes que en las plantas se producen en muy bajas cantidades, son costosos para producirlos sintéticamente en el laboratorio o sencillamente no se pueden producir (Zavala et al., 2009; Ramírez et al., 2013). Debido a esto, se han llevado a cabo trabajos de investigación donde, al igual que el presente estudio, se logró mejorar las características morfológicas de las plantas y la concentración de metabolitos secundarios como consecuencia de la inducción artificial de la poliploidía. Tal es caso de un clon de Taxus chinensis que al ser sometido a un proceso de inducción de poliploidía con colchicina, sincronización celular y selección de plantas tetraploides durante siete ciclos de multiplicación in vitro, se logró el 62,3 \% de plantas tetraploides, las cuales produjeron mayor cantidad del metabolito taxol que las plantas control (Li et al., 2008).

Así mismo, Omidbaigi et al. (2010) obtuvieron plantas autotetraploides de Dracocephalum moldavica que incrementaron la producción de aceites esenciales en un 27,5 \%. En Artemisia annua la duplicación cromosómica no sólo trajo como consecuencia el aumento en la concentración de artemisina, sino que también las plantas presentaron mayor peso en comparación a los diploides (De Jesús y Weathers, 2003). Otro ejemplo de mejoramiento en la producción de metabolitos secundarios con poliploidización es el caso del metabolito diosgenina en Dioscorea zingiberensis (Huang et al., 2010), en la cual se logró obtener tetraploides estables que incrementaron los contenidos de sus metabolitos secundarios, entre éstos la vitamina $\mathrm{C}$, y modificar otras características relacionadas con la calidad del fruto que mejoraron su rendimiento.

Molero et al. (2016) determinaron la concentración y producción de aloína en las mismas poblaciones estudiadas en el presente trabajo, pero en condiciones naturales y ploidía de $2 n=14$ cromosomas, y encontraron que las plantas que se destacaron por tener los máximos valores fueron las provenientes de las poblaciones de Cumarebo y Caramón. Al llevar estas plantas a condiciones in vitro, sus características morfológicas, valores de peso seco, concentración y producción de aloína fueron similares, pero 
luego de la duplicación cromosómica, las mismas mediciones no mostraron el mismo incremento en comparación a la respuesta de otras poblaciones, tales como Carazao y Adaure. Una situación similar se presentó en el estudio actual en donde las respuestas de las plantas no tuvieron la misma magnitud entre las diferentes poblaciones, como ya se señaló con anterioridad.

En este punto es oportuno discutir acerca de los factores que pueden influir en la respuesta diferencial intrapoblacional de las plantas que pertenecen a una misma especie, en cuanto a las características morfológicas y producción de metabolitos secundarios. Autores como Imery, (2007), Hazrati et al. (2012) y Martínez et al. (2013) han comentado que las variación entre las poblaciones puede deberse a los factores ambientales como estrés de las zonas áridas, altas temperaturas, elevada radiación solar, escasez de humedad y bajo contenido de nutrientes en el suelo, intensidad de luz UV y presencia de patógenos; pero en condiciones in vitro, de donde proceden las plantas del presente estudio, estas causales quedarían automáticamente eliminadas porque todas las plantas fueron sometidas al mismo suministro de nutrientes, la misma humedad y disponibilidad de luz, temperatura y salubridad.

Un segundo factor, que podría explicar esta diferencia intrapoblacional es la estructura genética de cada población de origen. Sing et al. (2009) y Jayakrishna et al. (2011) destacan que la propiedad de las plantas de sábila de producir sus metabolitos secundarios está determinada por su genoma, lo que hace que una planta pueda producir mayor cantidad y variedad de productos que otra. De igual manera, Sepúlveda et al. (2003) indican que los cambios en genes específicos que participan en la síntesis de las enzimas involucradas en la producción de los metabolitos secundarios, donde se incluye la aloína, pudieron llevarse a efecto en función al proceso de adaptación de las plantas a su medio. Otros estudios han evidenciado modificaciones en los genes que participan en la síntesis de metabolitos secundarios implicados en la respuesta sistémica de la planta ante heridas y ataques de patógenos (Ryan, 2000). Considerando lo anteriormente expuesto y analizando las diferentes condiciones climáticas de donde provienen las plantas de cada población, se tiene que Carazao y Adaure proceden es de una zona con clima equivalente al de un monte espinosotropical; Cumarebo, Tamare, Mayales y Puertos de Altagracia de un clima correspondiente a un bosque muy seco tropical, y Caramón del clima de un bosque seco premontano, según la clasificación de Ewel y Madriz (1976). En estas condiciones, es posible que se haya generado una respuesta genética para adaptarse a estas condiciones adversas, que se habría traducido en la modificación de los genes implicados en la producción de sus metabolitos secundarios y que se manifestarían de manera diferencial cuando las plantas fueron transferidas a condiciones de laboratorio.

Según Ewel y Madriz (1976), las características climáticas de un monte espinoso tropical se corresponden con altitudes de 0-200 msnm, temperatura superior a $25 \quad{ }^{\circ} \mathrm{C}$ y precipitación entre 250 y $500 \mathrm{~mm}$ anuales, con alta radiación solar; el bosque muy seco tropical posee temperaturas entre 23 y $29{ }^{\circ} \mathrm{C}$, y una precipitación de 500-1000 mm; el bosque seco premontano posee altitudes de $500-1500 \mathrm{msnm}$, temperaturas entre 18 y $24{ }^{\circ} \mathrm{C}$ y precipitación de $550-1100 \mathrm{~mm}$, es decir, a medida que se avanza en esta clasificación disminuye la temperatura y aumenta la precipitación. Se destaca, entonces, que las plantas de mejor respuesta (Carazao y Adaure) provienen de una zona con clima muy cálido y seco.

De acuerdo con la información proveniente del Cuadro 3, sobre la concentración y producción de aloína en las plantas control, debería esperarse que los altos valores encontrados en las poblaciones de Carazao y Caramón se mantuvieran luego del proceso de duplicación cromosómica. Sin embargo, esto no ocurrió, ya que además de destacarse las procedentes de Carazao, también resaltaron las procedentes de Adaure como las plantas que presentaron mayor concentración y producción de aloina. Al respecto, Omidbaigi et al. (2010) y Ramírez et al. (2013) indican que los efectos fisiológicos de la inducción de la poliploidía no son predecibles y las respuestas son a menudo específicas de cada especie o población de acuerdo con su estructura genética, ya que la duplicación en el número de cromosomas de una planta puede producir cambios en la actividad enzimática, afectando las vías de biosíntesis de metabolitos primarios y secundarios. Garro et al. 
(2008) confirman esta idea al detectar que la concentración de acemanan en los callos embriogénicos de $A$. vera tratados con colchicina fue menor con respecto a la concentración encontrada en las hojas frescas.

Otras investigaciones han logrado aumentar la concentración y cantidad de aloína en las células del tejido de $A$. vera manipulando diferentes variables como intensidad de luz (Páez et al., 2000), concentración hormonal (Kumar et al., 2016), dosis de nitrógeno (Hazrati et al., 2012) e incidencia de luz UV (Martínez et al., 2013), pero los rendimientos encontrados no superan los valores logrados en esta investigación por duplicación cromosómica principalmente en las plantas de las poblaciones Carazao y Adaure.

Muchos investigadores han manifestado la necesidad de seleccionar genotipos élites de $A$. vera (Imery y Cequea, 2001; Rathore et al., 2011) que presenten altas tasas de propagación y altos rendimientos de los productos y subproductos que se extraen de ella. Atendiendo a esta necesidad y considerando los resultados de esta investigación, se recomiendan las plantas poliploides de las poblaciones Carazao y Adaure para la extracción de aloína a nivel industrial. La selección de estas plantas con altas concentraciones de aloína, resulta atractivo tanto para programas de mejoramiento genético, como para ser utilizadas comercialmente, cuando su uso esté destinado a la industria manufacturera de cosméticos y fármacos.

\section{CONCLUSIONES}

A saber, este es el primer reporte sobre los efectos de la inducción artificial de la poliploidía en la producción de aloína en Aloe vera. Los nuevos conocimientos ponen de manifiesto el potencial de la poliploidía inducida como un medio rápido para mejorar la producción de metabolitos secundarios en plantas de interés económico. La comparación de los resultados entre las plantas control y poliploides indican que el aumento en el nivel de ploidía causó un incremento en la longitud foliar, número y ancho de hojas, peso seco, concentración y producción de aloína. De igual manera, puso de manifiesto que existen diferencias entre las poblaciones de sábila en respuesta a la duplicación cromosómica, siendo en este caso las plantas de las poblaciones de Carazao y Adaure las que tuvieron mejor respuesta en cuanto a concentración y producción de aloína. La explotación de estos nuevos genotipos podría mejorar el cultivo de la sábila a nivel comercial e industrial.

\section{AGRADECIMIENTOS}

$\mathrm{Al}$ Consejo de Desarrollo Científico y Humanístico de la Universidad del Zulia, Venezuela (CONDES-LUZ) por el financiamiento de esta investigación a través del proyecto CC0622-10, al Centro de Investigaciones Biológicas de la Facultad de Humanidades y Educación y al Laboratorio de Biotecnología de la Facultad de Agronomía de la misma universidad.

\section{LITERATURA CITADA}

1. Cardarelli, M., Y. Rouphaelb, M. Pellizzonic, G. Collad y L. Lucinic. 2017. Profile of bioactive secondary metabolites and antioxidant capacity of leaf exudates from eighteen Aloe species. Industrial Crops \& Products 108: 44-5.

2. Cheng, S., Y. Zong y X. Wang. 2019. SubGenome Polyploidization Effects on Metabolomic Signatures in Triploid Hybrids of Populus. Forests 10: 1091-1103.

3. Corneillie, S., N. De Storme, R. Van Acker, J. Fangel, M. De Bruyne, R. De Rycke et al. 2019. Polyploidy Affects Plant Growth and Alters Cell Wall Composition. Plant Physiology 179: 74-87.

4. Cubero, J. 2003. Introducción a la Mejora Genética Vegetal. Editorial Mundi-Prensa. Madrid.

5. De Jesús-González P. y J. Weathers. 2003. Tetraploid Artemisia annua hairy roots produce more artemisinin than diploids. Plant Cell Reports 21: 809-813.

6. Doyle, J. y J. Coate. 2019. Polyploidy, the nucleotype, and novelty: the impact of genome doubling on the biology of the cell. Inter. J. Plant Science 180 (1):1-52.

7. Ewel, J. y A. Madriz. 1976. Zonas de vida en Venezuela. Memorias explicativas sobre el mapa ecológico. Ministerio de Agricultura y Cría. Caracas. 264 p.

8. Garro-Monge G., A. Gatica-Arias y M. 
Valdez-Melara. 2008. Somatic embryogenesis, plant regeneration and acemannan detection in aloe (Aloe barbadensis Mill). Agronomía Costarricense 32: 41-52.

9. Hazrati S, T. Zeinolabedin, Z. Tahmasebi, y A. Baba A. 2012. Enhancing yield and aloin concentration of Aloe vera plants by simultaneous application of $\mathrm{N}$ and benzyladenine. Journal of Medicinal Plants Research 6(10): 1834-1841.

10.Huang, H., S. Gao, L. Chen y K. Wei. 2010. In vitro tetraploid induction and generation of tetraploids from mixoploids in Dioscorea zingiberensis. Pharmacognosy Magazine 6(21): 51-56.

11.Imery J. y H. Cequea. 2001. Colchicine-induce auto-tetraploid in Aloe vera L. Cytologia 66: 406-4012.

12.Imery, J. 2007. Inestabilidad cariológica durante la formación de células madre del polen en Aloe vera (Aloaceae). Revista Biología Tropical 55(3-4): 805-813.

13.Jayakrishna, C., C. Karthik, S. Barathi, D. Kamalanathan y A. Indra 2011. In vitro propagation of Aloe barbadensis Miller, a miracle herb. Research in Plant Biology 1: 2226.

14.Kumar D., S. Paridab y A. Dey. 2016. Comparative HPTLC analysis of bioactive marker barbaloin fromin vitro and naturally grown Aloe vera. Revista Brasileira de Farmacognosia 26: 161-167.

15.Li C., S. Fu, S. Wang, M. Li y L. Yu. 2008. Tetraploid induction of in vitro Taxus chinensis cell. Guihaia 1: 487-790.

16. Manzoor, A., T. Ahmad, M. Ajmal, I. Ahmad y C. Silvestri. 2019. Studies on colchicine induced chromosome doubling for enhancement of quality traits in ornamental plants. Plants 8(194): 1-16.

17.Martínez D., F. Guillén, H. Pérez, S. Castillo, M. Serrano, P. Zapata y D. Valero. 2013. Is it possible to increase the aloin content of Aloe vera by the use of ultraviolet light?. Agric. Food Chemistry 61(9): 2165-2170.

18. Martínez, W., A. Parody, M. Vélez, L. Paternina y R. Espitia. 2017. Optimización del proceso de eliminación de aloína del gel fileteado mecánico de Aloe vera concentrado
10X. Revista Cumbres 3(2): 9-16.

19. Molero T, y A. Matos. 2008. Efectos de la inducción artificial de la poliploidía en plantas de Aloe vera (L.) Burm.f. Boletín del Centro de Investigaciones Biológicas 42: 111-133.

20.Molero T, G. Ettiene y M. Viloria. 2016. Determinación de aloína en poblaciones de Aloe vera L. (= Aloe barbadensis M.) del occidente de Venezuela. Multiciencias 16(2): 143-152.

21.Molero T, M. Viloria y E. Viloria. 2018. Inducción de poliploidía con colchicina en vitroplantas de Aloe vera (L.). Revista Colombiana de Biotecnología 20(1): 108-116.

22. Omidbaigi R, S. Yavari, M. Hassani y S. Yavari. 2010. Induction of autotetraploidy in dragonhead (Dracocephalum moldavica L.) by colchicine treatment. Journal of Fruit and Ornamental Plant Research 18(1): 23-35.

23.Páez A, M. Gebre, M. González y T. Tschaplinski_ 2000. Growth, soluble carbohydrates and aloin concentration of Aloe vera plants exposed to three irradiance levels. Environment Experimental Botanical 44(2): 133-139.

24.Pandey, A. y S. Singh. 2016. Aloe Vera: A systematic review of its industrial and ethnomedicinal efficacy. International Journal of Pharmaceutical Research \& Allied Sciences 5(1): 21-33.

25.Park, M., J. Park, N. Young, Y. Geun, Y. Seok, J. Gyun, K. Ho y S. Ki. 1998. Analysis of 13 phenolic compounds in Aloe species by high performance liquid chromatography. Phytochemical Analysis 9: 186-191.

26.Pierce, B. 2009. Genética. Un Enfoque conceptual. Editorial Médica Panamericana. Madrid. pp. 255-258.

27.Piña H. y L. Chirino. 2008. Mercado de la sábila (Aloe vera L.) en el estado Falcón. Revista Facultad Agronomía (LUZ) 25: 364392.

28.Ramírez, F., V. Robledo, R. Foroughbakhch, A. Benavides, M. Alvarado y D. Quistian. 2013. Caracterización de tetraploides y formación de híbridos triploides en tomate de cáscara. Ciencia UANL 16 (64): 55-66.

29.Rathore M., J. Chikara y N. Shekhawat. 2011. 
Plantlet regeneration from callus cultures of selected genotypes of Aloe vera L.- An ancient plant for moderm herbal industries. Applied Biochemistry Biotechology 163: 860-868.

30.Ren Q., S. Li, M. Yao y B. Wang. 2007. Studies on induction of autotetraploid of Aloe vera L. Acta Agriculturae Boreali-Sinica 22: 136-138.

31.Ryan C. 2000. The systemin signaling pathway, differential activation of plant defensive genes. Biochimica et Biophysica Acta (BBA) - Protein Structure and Molecular Enzymology 1477(1-2): 112-121.

32.Sepúlveda-Jiménes F., H. Porta y M. Rocha. 2003. La participación de los metabolitos secundarios en la defensa de las plantas. Revista Mexicana de Fitopatología 21: 355-363.

33.Sing M., M. Rathore, D. Panwar, J. Rathore, H. Dagla y N. Shekhawat. 2009. Micropropagation of Selected Genotype of Aloe vera L. An Ancient Plant for Modern Industry. Journal of Sustainable Forestry 28:
935-950.

34.Tan, F., M. Zhang, K. Xie, Y. Fan, X. Song, R. Wang, X. Wu, H. Zhang y W. Guo. 2019. Polyploidy remodels fruit metabolism by modifying carbon source utilization and metabolic flux in Ponkan mandarin (Citrus reticulata Blanco). Plant Science 289: 1-12.

35.Wang L, S. Zeng, Z. Li y Z. Gu. 2001. A preliminary study on the polyloid induction and variation of Aloe vera. Acta Botanica Yunnanica 23: 493-496.

36.Zabala, M., M. Angarita, A. Aguirre, J. Restrepo y C. Montoya. 2009. Estrategias para incrementar la producción de metabolitos secundarios en cultivos de células vegetales. Rev. Fac. Nac. Agron. Medellín 62(1): 48814895.

37.Zanmaria, S. L. 2016. Melhoramento de artemisia annua L.: Indução de poliploidia expressão gênica e seleção de genótipos de alto rendimento. Tesis. Universidad Tecnológica Federal de Paraná. Brasil. 114 p. 